\title{
LES POLONAIS EN BIÉLORUSSIE ET EN UKRAINE
}

La plupart des territoires de la Biélorussie et de l'Ukraine actuelles appartenaient jusqu'à la fin du XVIII ${ }^{\mathrm{e}} \mathrm{s}$. à la République des Deux Nations: du Royaume de Pologne et du Grand-Duché de Lituanie. Les Polonais y constituaient une couche privilégiée de nobles et de grands propriétaires terriens. Ils étaient aussi nombreux dans les villes. Les Biélorusses et les Ukrainiens peuplaient surtout les villages.

Après le $1^{\text {er }}$ et le $2^{\mathrm{e}}$ partage de la Pologne en 1772 et 1793 , la majorité de ces territoires a été englobée par la Russie tsariste; seule la partie occidentale de l'Ukraine (la Galicie orientale) a été rattachée à l'Autriche. Les terres administrées par la Russie furent soumises à une russification intense. La répression frappa durement les Polonais après l'insurrection de 1831, et surtout après celle de 1863. De nombreuses arrestations et déportations en Sibérie et à l'intérieur de la Russie ont provoqué une diminution du nombre de Polonais.

Selon le recensement de la population effectué par les autorités tsaristes en 1897, les cinq gouvernements faisant partie de la Biélorussie actuelle comptaient 6424000 habitants, dont 839000 Polonais (13.1\% de l'ensemble de la population). Ils habitaient surtout les gouvernements occidentaux, comme lindique le tableau suivant (ou, en ce qui concerne les gouvernements de Vilnius et Grodno, seuls les territoires faisant partie de la Biélorussie actuelle ont été pris en compte):

\begin{tabular}{|l|c|c|}
\hline Gouvernement & \multicolumn{2}{|c|}{$\begin{array}{c}\text { Polonais (en milliers) et leur pourcentage } \\
\text { par rapport à l'ensemble de la population en 1897 }\end{array}$} \\
\hline Vilnius & 365,5 & $(43,0 \%)$ \\
Grodno & 148,9 & $(13,7 \%)$ \\
Minsk & 215,7 & $(10,1 \%)$ \\
Vitebsk & 63,4 & $(9,3 \%)$ \\
Mogilev & 45,4 & $(2,7 \%)$ \\
\hline
\end{tabular}

Sur le territoire de l'actuelle Ukraine, le recensement russe de 1897 concernait trois gouvernements, et le recensement autrichien de 1900 - la Galicie orientale et la Bukovine du Nord. Au total, sur 14037700 
habitants, il y avait 1942800 Polonais (13.8\%). En excluant les gouvernements orientaux où le nombre de Polonais était insignifiant, leur répartition était la suivante (en milliers et leur pourcentage par rapport à l'ensemble de la population):

\begin{tabular}{|l|r|r|}
\hline Gouvernement ou district & \\
\hline Volhynie (1897) & 291,2 & $(9,9 \%)$ \\
Podolie (1897) & 261,1 & $(8,8 \%)$ \\
Kiev (1897) & 103,9 & $(2,9 \%)$ \\
Galicie orientale (1900) & 1286,6 & $(30,8 \%)$ \\
Bukovine du Nord (1900) & 18,6 & $(4,5 \%)$ \\
\hline
\end{tabular}

Le pourcentage de Polonais était particulièrement élevé dans la ville de Lwow, où ils représentaient plus de $75 \%$ d'habitants - ainsi que dans quelques districts occidentaux (Lwow, Ternopol, Trembovla).

La reconstitution d'un État polonais indépendant en 1918 provoqua un mouvement de migration des Polonais de Russie vers la Pologne, surtout après la guerre polono-soviétique de 1920 et après l'établissement des frontières d'État en 1922. Le premier recensement de la population, effectué en URSS en 1926, a relevé 790000 Polonais, dont 476400 en Ukraine (1,6\% de la population) et 97500 en Biélorussie (2\%). Dans le recensement polonais de 1931 ont été considérés comme Polonais les habitants qui déclaraient le polonais comme leur langue maternelle. La religion était le deuxième critère; on admettait que le catholique était Polonais, l'orthodoxe ou le gréco-catholique - Biélorusse ou Ukrainien, éventuellement Russe. Les résultats de ces recensements étaient les suivants (Polonais en milliers et leur pourcentage par rapport à l'ensemble de la population):

\begin{tabular}{|l|c|c|}
\hline \multicolumn{2}{|c|}{ districts biélorusses (1926) } \\
\hline Minsk & 13,7 & $(2,5 \%)$ \\
Bobrouisk & 11,3 & $(2,1 \%)$ \\
Borisov & 16,2 & $(4,2 \%)$ \\
Vitebsk & 10,2 & $(1,7 \%)$ \\
Gomel & 6,5 & $(1,6 \%)$ \\
Kalinine & 0,7 & $(0,2 \%)$ \\
Mogilev & 5,6 & $(1,1 \%)$ \\
Mozyr & 8,9 & $(2,7 \%)$ \\
Orcha & 6,4 & $(1,5 \%)$ \\
Polotsk & 8,8 & $(2,7 \%)$ \\
Sluzk & 3,9 & $(1,3 \%)$ \\
Retsisz & 5,3 & $(2,1 \%)$ \\
\hline \multicolumn{1}{|c|}{ voíevodies polonaises (1931, les parties appartenant actuellement à la Biélorussie) } \\
\hline Vilnius & 383,6 & $(52,5 \%)$ \\
Novogrudok & 553,8 & $(52,4 \%)$ \\
Bialystok & 184,2 & $(47,9 \%)$ \\
Polésie & 157,3 & $(15,2 \%)$ \\
\hline
\end{tabular}


Dans la Biélorussie soviétique la population polonaise était importante surtout à l'Ouest. La plus grande concentration se trouvait aux environs de Koidanov (Dzerjinsk), au Sud-Ouest de Minsk, où en 1930 les Polonais constituaient $80 \%$ d'habitants. En 1932 y fut créée une région polonaise autonome, liquidée en 1937, dans le cadre de la répression menée par les autorités soviétiques contre la population polonaise. Le nombre de Polonais tomba de 119900 en 1937 a 58400 en 1939.

Dans la partie polonaise de l'actuelle Biélorussie les plus grands foyers de peuplement polonais se trouvaient dans les districts situés à l'Ouest.

Dans la même période, en Ukraine Soviétique les Polonais habitaient surtout les régions de Polésie et de la rive droite du Dniepr; dans certains districts, ils constituaient plus de $10 \%$ de la population. Là aussi, à Marchlevsk (Dolbysh, $70 \%$ de Polonais), au Sud-Ouest de Jitomir en Volhynie, une région polonaise autonome fut créée en 1925; on en prévoyait une autre à Khmelnitski, en Podolie. La répression des années trente conduisit à la liquidation de la région; celle de Khmelnitski n'a jamais vu le jour. De nombreux Polonais furent déportés au Kazakhstan et en Sibérie.

Dans la partie polonaise de l'actuelle Ukraine les Polonais étaient bien sûr beaucoup plus nombreux et dans plusieurs districts représentaient plus de la moitié de la population $(63,5 \%)$ dans la ville de Lvov. Dans l'ensemble, dans les territoires de l'actuelle Ukraine la répartition était la suivante: (Polonais en milliers et leur pourcentage par rapport au total de la population, par région en 1926 ou voïévodie en 1931):

\begin{tabular}{|l|r|r|}
\hline Polésie & 128,9 & $(4,4 \%)$ \\
Rive droite du Dniepr & 282,5 & $(3,1 \%)$ \\
Rive gauche du Dniepr & 12,2 & $(0,2 \%)$ \\
Moldavie & 4,6 & $(0,8 \%)$ \\
Steppes & 34,5 & $(0,6 \%)$ \\
Zaporoje & 9,2 & $(0,4 \%)$ \\
Volhynie & 396,2 & $(17,3 \%)$ \\
Lvov (partie act. ukrainienne) & 706,0 & $(46,1 \%)$ \\
Ternopol & 789,0 & $(49,3 \%)$ \\
Stanislavov (Ivano-Frankovsk) & 358,4 & $(22,5 \%)$ \\
\hline
\end{tabular}

Après l'invasion des troupes soviétiques le 17 septembre 1939 des confins orientaux de la Pologne, ces territoires furent incorporés à la Biélorussie et à l'Ukraine, à l'exception d'une partie de la voiévodie de Vilnius, rattachée à la Lituanie. C'est ainsi que le nombre de Polonais habitant la Biélorussie et l'Ukraine augmenta considérablement. Dans les terres annexées par l'Union Soviétique et faisant partie de la Biélorussie et l'Ukraine actuelles 3900000 personnes ( $36 \%$ de la population) déclaraient la langue polonaise comme langue maternelle. Environ 200000 Polonais furent déportés de la Biélorussie à l'intérieur de la Russie et au Kazakhstan; 
environ 80000 partirent pour la Pologne centrale. De l'Ukraine Occidentale env. 230000 furent déportés surtout au Kazakhstan, en Sibérie et en Extrême-Orient soviétique. D'après le recensement allemand effectué en 1942, il y avait 1000000 de Polonais en Galicie orientale et 305000 en Volhynie; le nombre de ces derniers est tombé à 200000 en 1943, surtout en raison de la répression des nationalistes ukrainiens. Après la guerre et l'établissement des frontières entre la Pologne et l'Union Soviétique, on entreprit de déplacer la population polonaise de la Biélorussie et l'Ukraine en Pologne - et de la population biélorusse et ukrainienne de Pologne en Union Soviétique. Seuls ceux qui possédaient la citoyenneté polonaise avant 1939 pouvaient se rendre en Pologne. En 1942-45, dans le cadre de cette "action de rapatriement", 34000 Biélorusses partirent pour la Biélorussie, 274200 Polonais - pour la Pologne. La majeure partie de l'intelligentsia quitta ces confins orientaux. Dans la même période, 810500 Polonais quittèrent l'Ukraine (dont 352200 le district de Lvov, 229700 - celui de Ternopol), où s'établirent 482100 Ukrainiens venus de Pologne.

En Union Soviétique, le premier recensement de population après la $2^{\mathbf{e}}$ Guerre mondiale fut effectué en 1959, les suivants en 1970,1979 et 1989 . Les données sont comparables, car elles concernent les mêmes unités spatiales et furent recueillies selon les mêmes principes. Le nombre de Polonais était toujours sous-estimé, pour des raisons politiques: les autorités ne tenaient pas à faire apparaître l'existence de groupes nationaux. La population polonaise a été soumise à une assimilation, favorisée par le manque d'écoles polonaises et d'églises catholiques. De nombreux Polonais ne reconnaissaient pas leur polonité, de peur de répression. Ainsi, malgré l'accroissement naturel et bien qu'il n'y ait pratiquement pas d'émigration, le nombre de Polonais diminue ou n'augmente que très peu. Ainsi, en Biélorussie l'évolution était la suivante (les Polonais en milliers et leur pourcentage par rapport à l'ensemble de la population par district):

\begin{tabular}{|lrrrr|}
\hline & \multicolumn{1}{c}{1959} & \multicolumn{1}{c|}{1970} & \multicolumn{1}{c|}{1979} & \multicolumn{1}{c|}{1989} \\
\hline Briest & $42,1(3,5 \%)$ & $\mathbf{3 2 , 5}(2,5 \%)$ & $33,9(2,5 \%)$ & $31,7(2,2 \%)$ \\
Vitebsk & $\mathbf{8 3 , 8}(6,6 \%)$ & $24,6(1,5 \%)$ & $17,3(1,2 \%)$ & $25,3(1,8 \%)$ \\
Gomel & $7,2(0,5 \%)$ & $4,8(0,3 \%)$ & $4,7(0,3 \%)$ & $4,5(0,3 \%)$ \\
Grodno & $332,3(30,8 \%)$ & $276,5(24,7 \%)$ & $300,2(26,6 \%)$ & $300,8(25,9 \%)$ \\
Minsk-ville & $5,6(1,1 \%)$ & $9,4(1,0 \%)$ & $14,6(1,1 \%)$ & $18,5(1,2 \%)$ \\
Minsk & $64,4(4,4 \%)$ & $31,3(2,0 \%)$ & $28,7(1,8 \%)$ & $33,2(2,1 \%)$ \\
Mogilev & $3,5(0,3 \%)$ & $3,2(0,3 \%)$ & $3,8(0,3 \%)$ & $3,7(4,1 \%)$ \\
Biélorussie & $538,9(6,7 \%)$ & $382,6(4,3 \%)$ & $403,2(4,2 \%)$ & $417,7(4,1 \%)$ \\
\hline
\end{tabular}

Les Polonais y habitent surtout à l'Ouest, particulièrement dans le district de Grodno, ainsi qu'à Lida où l'on trouve des organisations, des journaux et des groupes folkloristiques polonais. 306 églises catholiques ont déjà été ouvertes en 1991; en 1992-93, dans 304 écoles 13950 enfants recevaient un enseignement en polonais. 


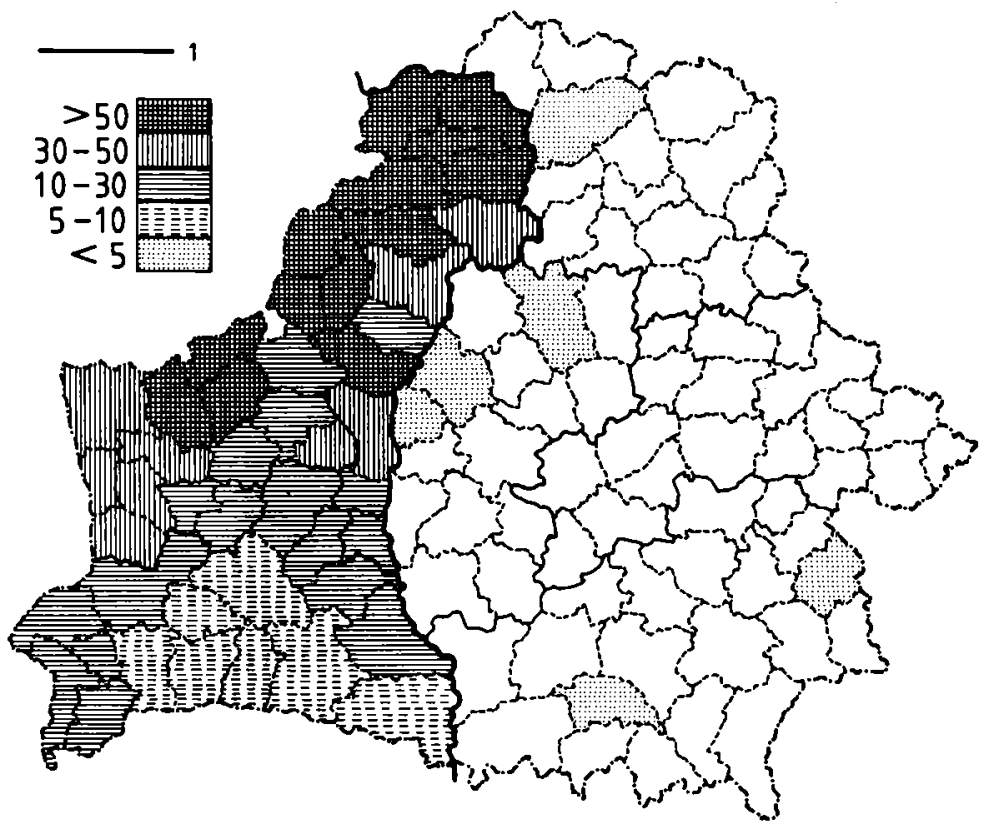

Fig. 1. La répartition des Polonais en Biélorussie, en 1939 (en \%) 1. frontière polono-soviétique

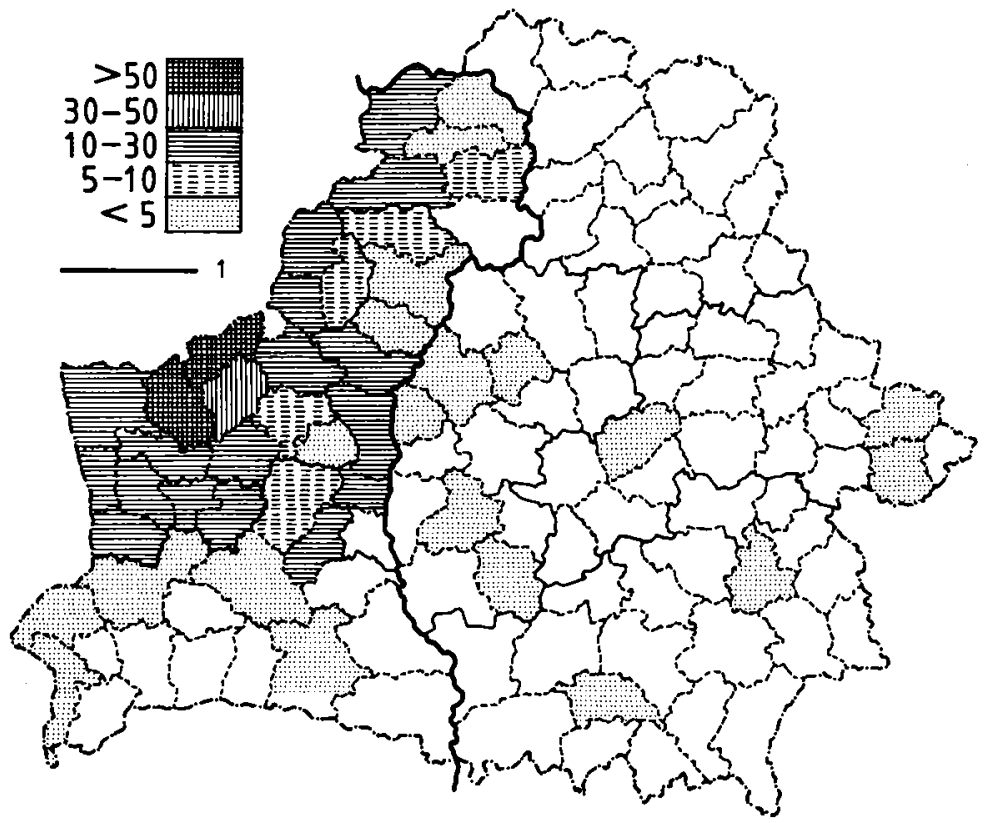

Fig. 2. La répartition des Polonais en Biélorussie, en 1989 (en \%) 1. frontière polono-soviétique en 1939 


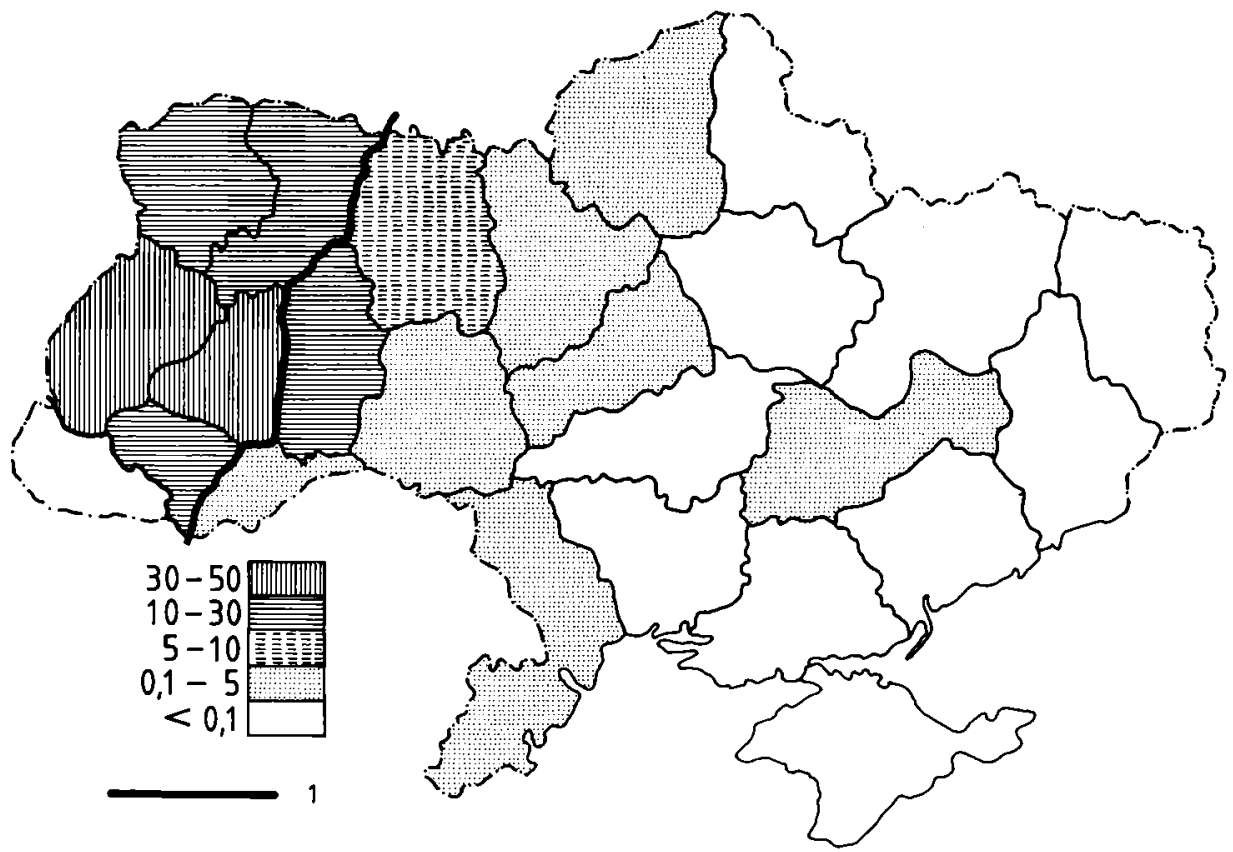

Fig. 3. La répartition des Polonais en Ukraine, en 1926/1931 (en \%)

1. frontière polono-soviétique

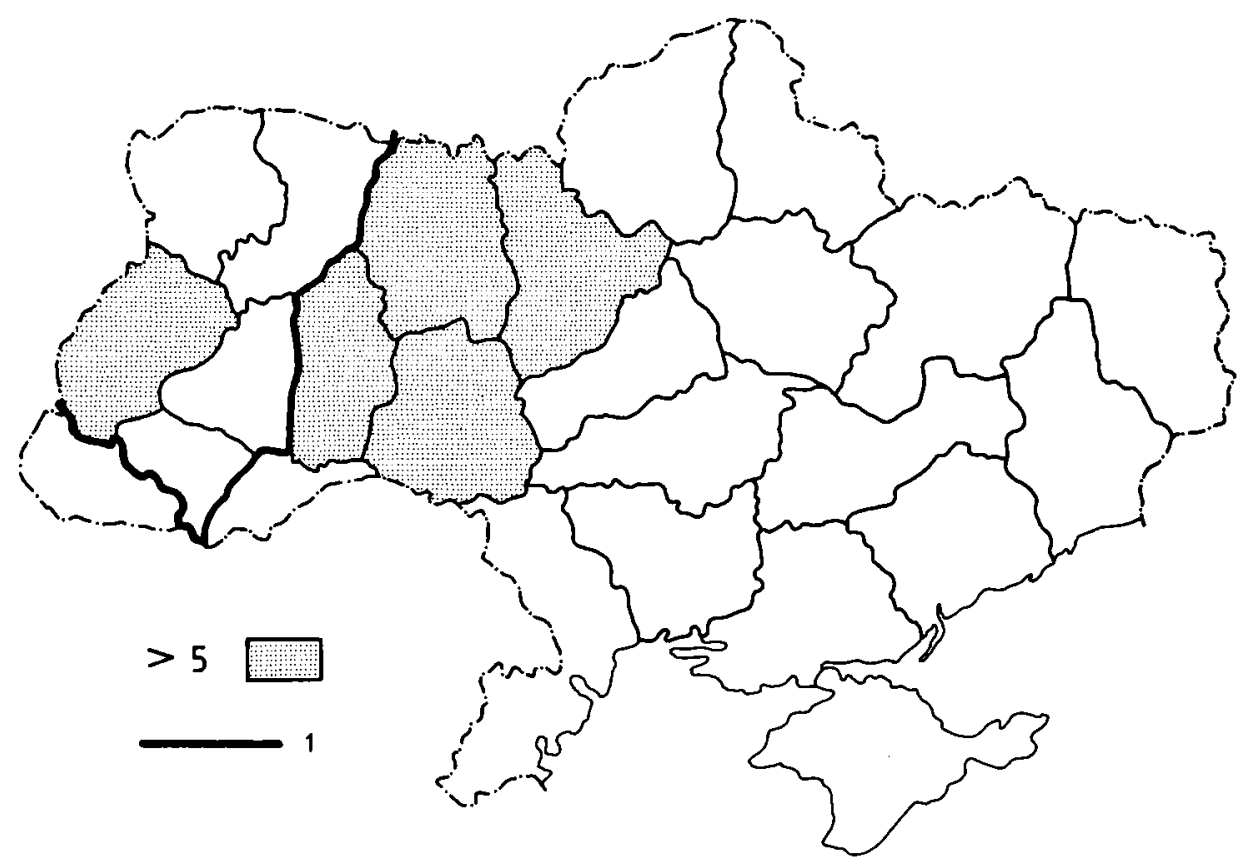

Fig. 4. La répartition des Polonais en Ukraine, en 1989 (en \%)

1. frontière polono-soviétique en 1939 
En Ukraine le nombre de Polonais diminue constamment, surtout à l'Ouest, où autrefois ils étaient le plus nombreux. Les foyers polonais se trouvent actuellement à Jitomir et Khmelnitski, donc dans les régions qui avant la guerre se trouvaient en URSS. Lvov, qui constituait le centre polonais le plus important dans les confins du Sud-Est, n'occupe que la $3^{e}$ place, à cause d'émigration évoquée plus haut. Les Polonais y vivent beaucoup plus dispersés qu'en Biélorussie. D'après les données fournies par les milieux polonais, done non officielles, à Lvov on compte de 10 à 13000 Polonais, à Kiev ils seraient 20000 , à Berditchov - 10 000, à Jitomir - de 60 à 100000 , à Vinnitsa - 40000 . Il est à noter que peu de Polonais déclarent le polonais comme leur langue maternelle: à Jitomir ils ne seraient que $0,6 \%$, à Lvov $-\mathbf{4 8} \%$. Une certaine renaissance est favorisée par l'église catholique. 82 prêtres, dont 61 venus de Pologne, exercent dans l'archevêché de Lvov qui compte 210 paroisses. A Tchernovtsy il y a 21 paroisses, en Volhynie - 10, à Rovno - 10, à Ivano-Frankovsk - 18, à Ternopol -55 . Selon les recensements, la situation évoluait de manière suivante (Polonais en milliers et leur pourcentage par rapport à l'ensemble de la population, par district):

\begin{tabular}{|lrcrccc|}
\multicolumn{2}{c}{1959} & \multicolumn{2}{c}{1970} & \multicolumn{2}{c}{1989} \\
\hline Kiev-ville & $\mathbf{8 , 5}$ & $(0,8 \%)$ & 9,7 & $(0,6 \%)$ & 10,4 & $(0,2 \%)$ \\
Khmelnitski & 70,1 & $(4,4 \%)$ & 52,6 & $(3,3 \%)$ & 36,7 & $(2,4 \%)$ \\
Tchernovtsy & 6,0 & $(0,8 \%)$ & 5,3 & $(0,6 \%)$ & $*$ & $*$ \\
Dniepropetrovsk & 6,1 & $(0,2 \%)$ & 6,3 & $(0,2 \%)$ & $*$ & $*$ \\
Ivano-Frankovsk & 10,4 & $(1,0 \%)$ & 6,5 & $(0,5 \%)$ & $*$ & $*$ \\
Kiev & 7,2 & $(0,4 \%)$ & 5,9 & $(0,3 \%)$ & $*$ & $*$ \\
Lvov & 59,1 & $(2,8 \%)$ & 41,5 & $(41,5 \%)$ & 26,9 & $(1,0 \%)$ \\
Rovno & 4,4 & $(0,5 \%)$ & 3,7 & $(0,4 \%)$ & $*$ & $*$ \\
Ternopol & 23,5 & $(2,2 \%)$ & 14,7 & $(1,3 \%)$ & $*$ & $*$ \\
Vinnitsa & 20,8 & $(1,0 \%)$ & 13,4 & $(0,1 \%)$ & 8,4 & $(0,4 \%)$ \\
Jitomir & 103,0 & $(6,4 \%)$ & 90,7 & $(5,6 \%)$ & 69,4 & $(4,5 \%)$ \\
Crimée & $*$ & $*$ & 6,0 & $(0,3 \%)$ & $*$ & $*$ \\
Lougansk & $*$ & $*$ & 4,6 & $(0,2 \%)$ & $*$ & $*$ \\
Kherson & $*$ & $*$ & 2,8 & $(0,3 \%)$ & $*$ & $*$ \\
Nikolaiev & $*$ & $*$ & 2,5 & $(0,2 \%)$ & $*$ & $*$ \\
Zaporoje & $*$ & $*$ & 2,2 & $(0,1 \%)$ & $*$ & $*$ \\
Ukraine & 363,3 & $(0,9 \%)$ & 295,1 & $(0,6 \%)$ & 219,2 & $(0,4 \%)$ \\
\hline
\end{tabular}

Ainsi, jusqu'en 1989, aussi bien en Biélorussie qu'en Ukraine le pourcentage de Polonais diminuait constamment. Leur situation s'est d'une part améliorée grâce au changement de régime, mais d'autre part les tendances nationalistes, parfois exagérées mais compréhensibles, se manifestant en Ukraine et dans les premières années de l'indépendance en Biélorussie, ne leur étaient pas favorables. 
